\title{
Bone texture modifications during bone regeneration and osteocyte cell-signaling changes in response to treatment with Teriparatide
}

\author{
Alberto Smargiassi, Marta Checchi, Francesco Cavani, Marzia Ferretti \& Carla \\ Palumbo
}

\section{Author affiliations}

Teriparatide is the active fragment (1-34) of the endogenous human parathyroid hormone (PTH). Studies showed that chronic administration of PTH results in decreased bone mass while intermittent exposure to PTH activates osteoblast bone deposition. Most of the structural studies published so far about the effect of Teriparatide focused their attention on the amount of newly-deposed bone without investigating the quality of the newly-formed bone tissue; moreover, most of the papers enhance the attention to the osteoblast's cellular and molecular involvement during the process of bone repair, ignoring the pivotal role that osteocytes play in the bone environment by means of the modulation of their signaling. During bone regeneration, after a preliminary formation of fibrous tissue with massive vascular proliferation, two different types of osteogenesis follow each other: firstly the process of Static Osteogenesis, which produces preliminary bony trabeculae with woven texture and, later, the process of Dynamic Osteogenesis, in which osteoblasts, driven by osteocytes, produce a more ordered and more mechanical valid bone. This work includes both in vivo and in vitro investigations: i) in vivo morphological analyses of qualitative repair of experimentally induced lesions in diaphysis of rats femurs and ii) in vitro study of the osteocyte cell-signaling, both in response to Teriparatide treatment, in order to deepen the knowledge of morphological and molecular events occurring in bone regeneration, with particular attention to the osteocyte signaling. The results obtained showed that the intermittent administration of Teriparatide in vivo anticipates the beginning of Dynamic Osteogenesis, which is characterized by the production of a more ordered and resistant bone. The molecular biology data, performed on an osteocyte immortalized cellular model (MLO-Y4) treated in vitro with the drug at different time points showed, by microarray and western blot analysis, different gene/protein expression involved in osteocyte signaling compared with the control condition. 